\title{
Metrology of Optical Communication Systems Using Error Vector Magnitude
}

\author{
Irshaad Fatadin \\ National Physical Laboratory, Teddington, UK \\ Email: irshaad.fatadin@npl.co.uk
}

How to cite this paper: Fatadin, I. (2021) Metrology of Optical Communication Systems Using Error Vector Magnitude. Journal of Applied Mathematics and Physics, 9, 2918-2926. https://doi.org/10.4236/jamp.2021.911185

Received: August 24, 2021

Accepted: November 20, 2021

Published: November 23, 2021

\begin{abstract}
Error vector magnitude (EVM) as a performance metric for $M$-ary quadrature amplitude modulation (QAM) formats in optical coherent systems is presented. It is shown that the calibrated BER, which would otherwise be under-estimated without the correction factor, can reliably monitor the performance of optical coherent systems near the target BER of $10^{-3}$ for quadrature phase shift keying (QPSK), 16-QAM, and 64-QAM employing carrier phase recovery with differential decoding to compensate for laser phase noise. The impact on the number of symbols used to estimate the BER from EVM analysis is also presented and compared to the BER obtained by error counting.
\end{abstract}

\section{Keywords}

Carrier Phase Recovery, Error Vector Magnitude (EVM), Quadrature Amplitude Modulation (QAM)

\section{Introduction}

Optical performance monitoring is an important aspect in the design of the next generation of optical communication systems [1]. Accurate measurement analysis needs to be developed to quantify the novel modulation schemes and to reliably predict the performance of optical coherent systems encoding information on both the amplitude and phase of the optical carrier for $M$-ary quadrature amplitude modulation (QAM) [2].

A number of performance metrics, such as bit error ratio (BER), eye diagram, Q-factor, Optical Signal to Noise Ratio (OSNR), and, more recently, error vector magnitude (EVM), can be used in optical communications to assess the quality of the transmitted signals [3] [4] [5]. BER is regarded as the most conclusive figure of merit compared to the other performance metrics for advanced modulation formats. However, estimating the BER in communication links requires a 
known pattern to be transmitted for continuous performance monitoring which can also be time-consuming if the BER is small. EVM is a promising alternative performance metric at a high signal-to-noise ratio (SNR) where a large number of symbols will otherwise be required for accurate error counting. EVM is a standard figure of merit to evaluate the quality of vector-modulated signals in wireless digital communication systems [6] and has also been applied to assess the performance of optical channels corrupted by amplified spontaneous emission (ASE) noise and to characterize optical $I-Q$ transmitters [3] [4]. EVM is better suited than BER counting for unknown symbol sequences and the established relationship between EVM and BER for data-aided reception [7] can also be applied to nondata-aided reception as noted in [3]. The relationship between EVM and BER is of particular interest for optical coherent systems operating near the forward error correction (FEC) limit.

In this paper, the estimation of BER from EVM analysis is presented for quadrature phase shift keying (QPSK), 16-QAM, and 64-QAM with carrier phase recovery and differential decoding to compensate for laser phase noise. It is shown that EVM can be an invaluable metrological tool to monitor the performance of optical communication links. Differential decoding is commonly employed with carrier phase recovery algorithms to solve the four-fold ambiguity associated with the recovered phase for square-QAM constellations and also to avoid performance degradation due to cycle slips [8] [9]. As such, the BER estimated from EVM in this work is compared to the actual BER expected with differential decoding. The BER derived from EVM analysis is demonstrated to be under-estimated following the compensation of laser phase noise for the different modulation formats. A correction factor is thus necessary to reliably estimate the BER from EVM and to accurately monitor the performance of optical coherent systems. Finally, the impact on the number of symbols used to estimate the BER from EVM analysis is also presented and compared to the BER obtained by error counting.

\section{Error Vector Magnitude and BER Estimation}

EVM is defined as the root-mean-square (rms) value of the difference between a collection of ideal transmitted symbols and the received symbols in the $I-Q$ plane. The error vector between the received symbol and the ideal symbol location is shown in Figure 1.

The rms EVM can be expressed as [3] [10]

$$
E V M_{r m s}=\sqrt{\frac{\frac{1}{N} \sum_{i=1}^{N}\left|E_{r, i}-E_{t, i}\right|^{2}}{P_{a}}},
$$

where $N$ is the number of symbols over which the EVM is estimated, $E_{r}$ is the received signal vector, $E_{t}$ is the ideal transmitted vector, and $P_{a}$ is the average ideal transmitted power for the chosen modulation. $E_{t}$ can either be a known reference pattern for data-aided reception or computed relative to the 


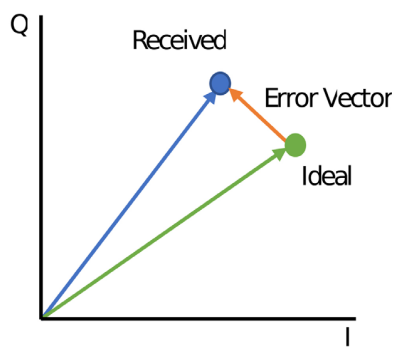

Figure 1. Error vector between the received symbol and the ideal symbol location.

closest symbol in the constellation for nondata-aided reception. For $M$-ary square QAM constellations and data-aided reception, where the EVM is caused by ASE noise, the BER can be estimated using [3] [7]

$$
B E R=F \cdot \frac{\left(1-M^{-1 / 2}\right)}{\frac{1}{2} \log _{2} M} \operatorname{erf}\left[\sqrt{\frac{3 / 2}{(M-1) E V M_{r m s}^{2}}}\right],
$$

where the differential coding penalty, $F$, is given as [11]

$$
F=1+\frac{\log _{2}(M)}{2(\sqrt{M}-1)} \text {. }
$$

For QPSK, 16-QAM, and 64-QAM, the values of $F$ can thus be set to 2, 1.67, and 1.43 , respectively, to include the expected differential decoding penalty for the different modulation formats.

Figure 2 shows the deviation of the EVM estimated for nondata-aided from data-aided reception for QPSK, 16-QAM, and 64-QAM using (1) with ASE noise included in the simulation. It can be seen that the EVM for nondata-aided reception is under-estimated at lower SNR values for the different modulation formats. The corresponding BER performance estimated from the EVM analysis is shown in Figure 3. The performance for data-aided reception was found to be in perfect agreement with the theoretical limit in the presence of ASE noise. At a target BER of $10^{-3}$, the BER can also be estimated from EVM without significant deviation from the expected value for nondata-aided reception. However, the deviation can be seen to increase at a BER of $10^{-2}$ for the higher-order modulation formats. For lower SNR, the EVM and, hence, the BER are under-estimated as the decision-directed symbols are mapped to the wrong constellation points for nondata-aided reception.

Next, the simulation was performed at a target BER of $10^{-3}$ in the presence of laser phase noise and differential encoding/decoding process. The BER estimated from EVM analysis is compared to the actual BER obtained by error counting in Figure 4 following the compensation of laser phase noise with differential decoding. The simulation was performed over 200,000 symbols. The carrier phase recovery was implemented using the feedforward blind phase search algorithm [8] which is applicable to arbitrary $M$-QAM constellations and suitable for real-time implementation with high linewidth tolerance. The number of test phases was set to 32 for QPSK/16-QAM and 64 for 64-QAM. The 


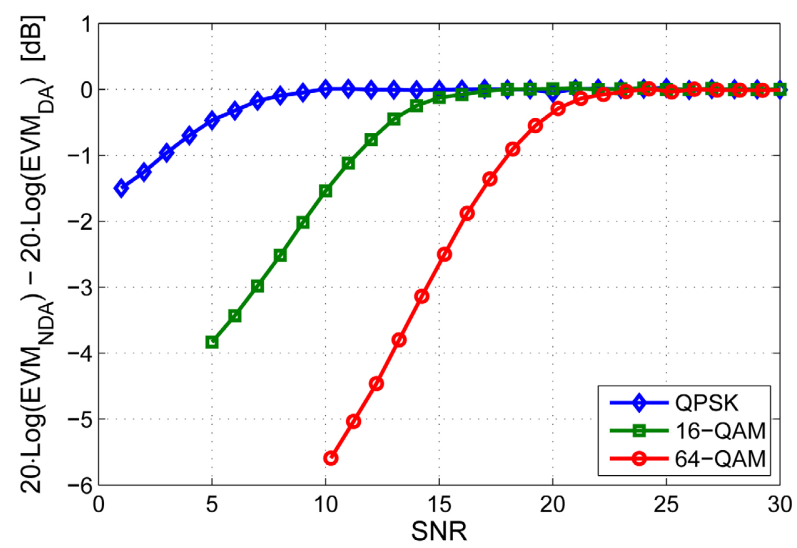

Figure 2. Deviation of estimated EVM for nondata-aided (NDA) from data-aided (DA) reception for QPSK, 16-QAM, and 64-QAM.

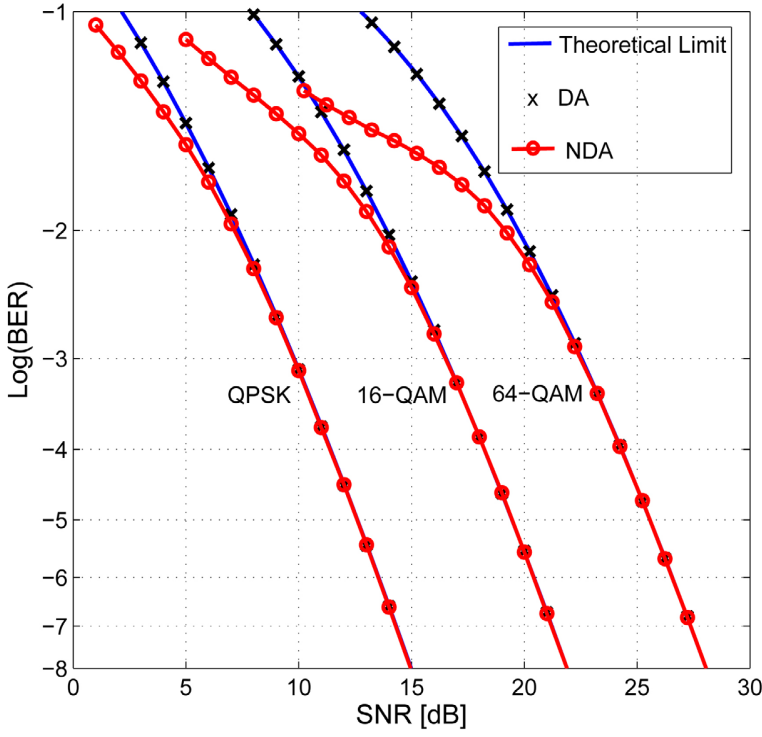

Figure 3. BER estimation from EVM analysis for data-aided (DA) and nondata-aided (NDA) reception for QPSK, 16-QAM, and 64-QAM.

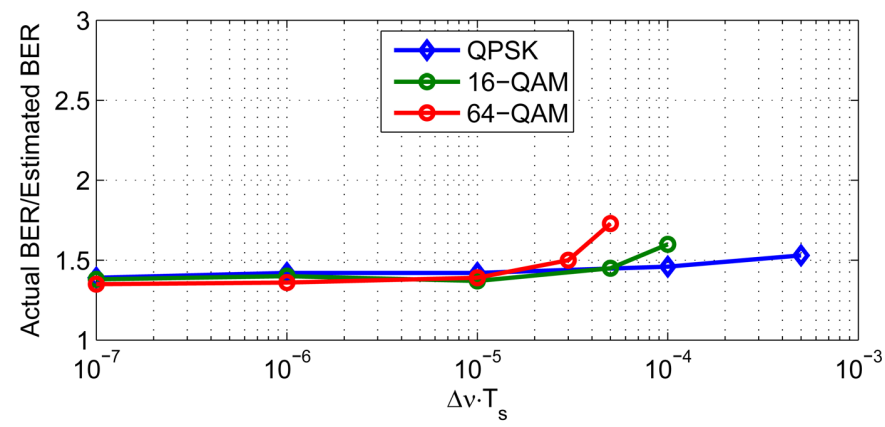

Figure 4. Ratio of actual BER to estimated BER for different values of $\Delta v \cdot T_{s}$ at the target BER of $10^{-3}$.

filter half width was set to 9 for the different modulation formats. The sensitivity penalty at the target BER of $10^{-3}$ was $<1 \mathrm{~dB}$ for the different modulation formats 
and also includes the coding penalty due to the differential encoding/decoding process. In the presence of laser phase noise and carrier phase recovery, the BER obtained from EVM analysis can be seen to be under-estimated in Figure 4 compared to the actual BER. A calibration of the estimated BER is thus necessary as discuss in the next section to reliably predict the performance of optical coherent systems with EVM analysis.

\section{BER Estimation with Correction Factor}

The results shown in Figure 4 reveal that the relationship (2) under-estimates the actual BER with the carrier phase recovery. The BER estimated from EVM analysis, $B E R_{E V M}$, can be calibrated using

$$
B E R_{\text {cal }}=k \cdot B E R_{E V M} \text {, }
$$

where $k$ is a correction factor to take into account the implementation penalty of the carrier phase recovery process in addition to the differential coding penalty included in (2). Figures 5(a)-(c) show the deviation, $\varepsilon$, defined as
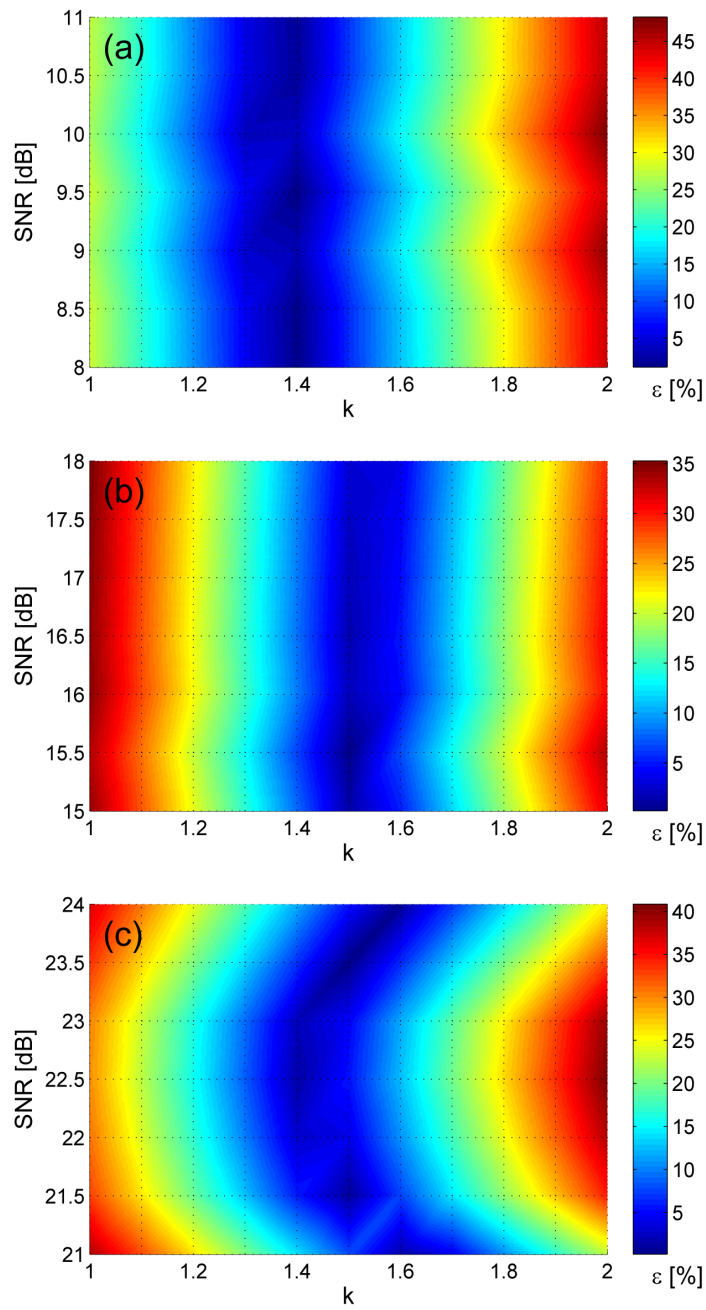

Figure 5. Deviation, $\varepsilon$, of estimated BER from actual BER with different values of correction factor, $k$, for (a) QPSK, (b) 16-QAM, and (c) 64-QAM. 


$$
\varepsilon=\left|1-\frac{k \cdot B E R_{E V M}}{B E R_{\text {actual }}}\right|,
$$

for QPSK, 16-QAM, and 64-QAM, respectively, near the target BER of $10^{-3}$. As it can be seen in Figure 5, an optimum value of $k$, where $\varepsilon=0$, can be chosen to calibrate the estimated BER from EVM analysis.

The calibrated and estimated BER from EVM analysis are compared with the actual BER in Figure 6 for optical performance monitoring near the target BER of $10^{-3}$. The insets show the recovered constellations at the BER of $10^{-3}$. Without
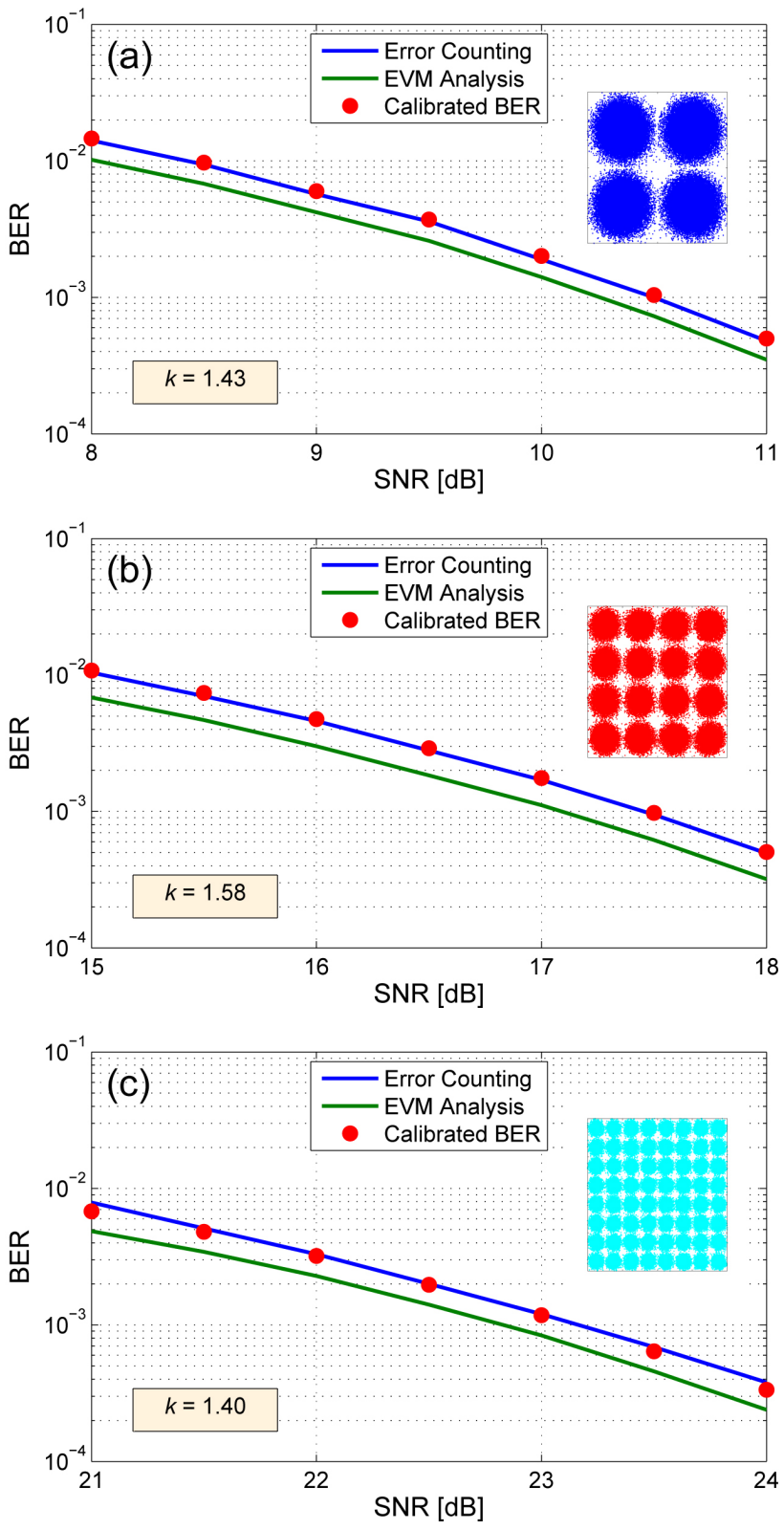

Figure 6. Performance of calibrated BER compared to the estimated BER from error counting and EVM analysis for (a) QPSK $\left(\Delta v \cdot T_{s}=10^{-4}\right)$, (b) 16-QAM $\left(\Delta v \cdot T_{s}=10^{-4}\right)$, and (c) 64-QAM $\left(\Delta v \cdot T_{\mathrm{s}}=10^{-5}\right)$. 
the correction factor, the relationship (2) under-estimates the BER at the different SNR as expected from the simulation results presented in Figure 4. However, the calibrated BER using (4) can be seen to be in close agreement with the actual BER obtained by error counting. The values of $k$ derived at the target BER of $10^{-3}$ were used for the simulation results shown in Figure 6. Although, the estimated BER from EVM analysis was found to be biased with the carrier phase recovery process, the above simulation results demonstrate that a correction factor can effectively be applied near the target BER for performance monitoring in optical coherent systems.

Finally, the impact on the number of symbols used to estimate the BER from EVM analysis was investigated and compared to the BER obtained by error counting. Figure 7 shows the impact of using different number of symbols on
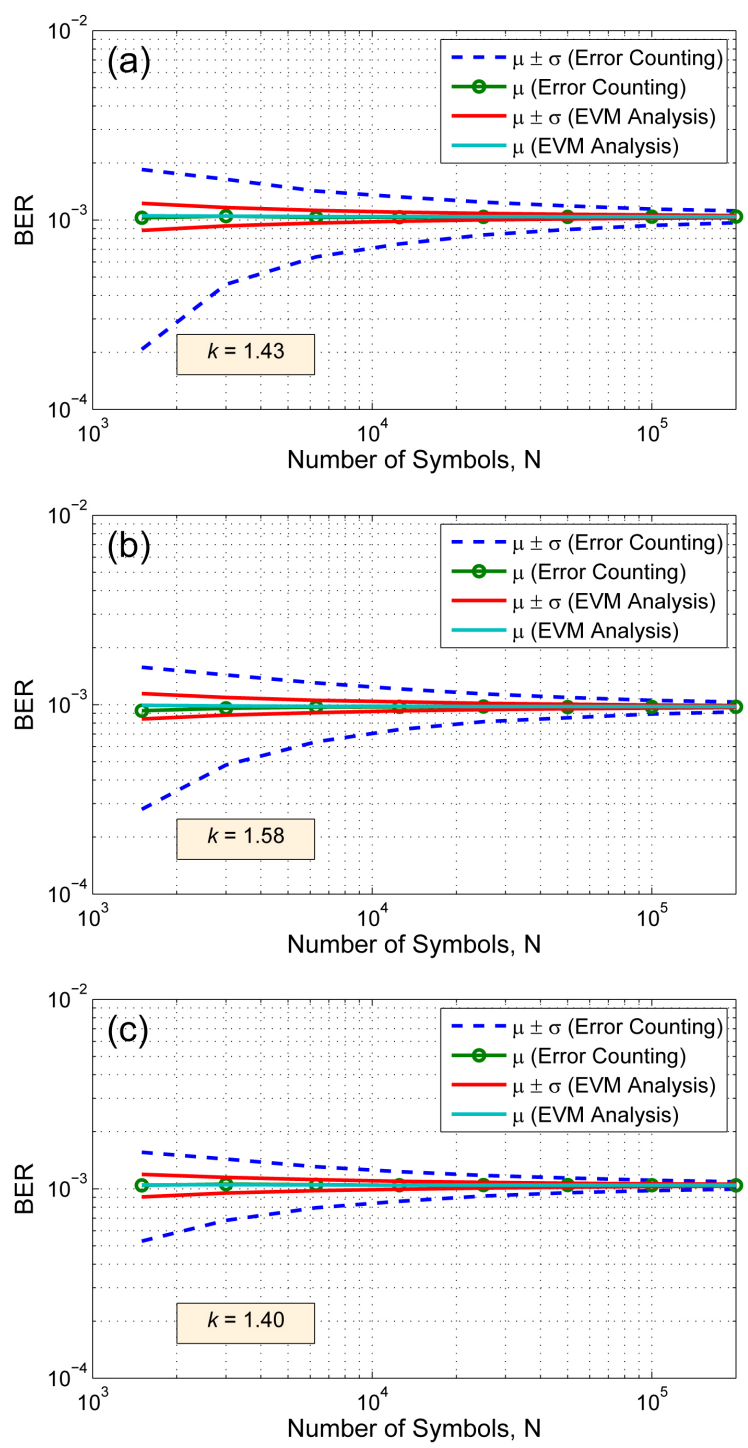

Figure 7. Impact on the number of symbols used to estimate the BER from error counting and EVM analysis for (a) QPSK (SNR = 10.5 dB), (b) 16-QAM (SNR = $17.5 \mathrm{~dB})$, and (c) 64-QAM $($ SNR = $23.1 \mathrm{~dB})$. 
the BER estimators. The mean values, $\mu$, over the 1000 trials can be seen to be similar for both techniques with the correction factor. However, from the curve bands, $\mu \pm \sigma$, where $\sigma$ is the standard deviation, it can be seen that an estimate with lower uncertainty can be achieved from EVM analysis compared to error counting, in particular, for low number of symbols. Thus, the calibrated BER can potentially be a useful figure of merit to reliably estimate the performance of optical coherent systems with EVM analysis as opposed to the large number of symbols that may be required for error counting to compute the actual BER. Moreover, the BER estimated from EVM analysis has the additional benefit that it can still be recovered for unknown symbol sequences transmitted over the optical network with low implementation complexity to evaluate the quality of advanced modulation formats.

\section{Conclusion}

Error vector magnitude (EVM), as a performance metric for multilevel modulation formats in optical coherent systems, has been presented. It is shown that the calibrated BER, which would otherwise be under-estimated without the correction factor, can reliably monitor the performance of optical coherent systems near the target BER of $10^{-3}$ for QPSK, 16-QAM, and 64-QAM employing carrier phase recovery with differential decoding to compensate for laser phase noise. The number of symbols required to estimate the BER from EVM analysis has also been presented and compared to the BER obtained by error counting.

\section{Acknowledgements}

This project 20SIP05 KTOC has received funding from the EMPIR programme co-financed by the Participating States and from the European Union's Horizon 2020 research and innovation programme, Funder ID: 10.13039/100014132. This work was also supported by the Department for Business, Energy \& Industrial Strategy (BEIS) at NPL.

\section{Conflicts of Interest}

The author declares no conflicts of interest regarding the publication of this paper.

\section{References}

[1] Tan, Q., Wang, Z., Yang, A. and Guo, P. (2019) Coherent Optical Sampling Based Method for Monitoring Optical Signal to Noise Ratio of High Speed Optical Fiber Communication Systems. 20197 th International Conference on Information, Communication and Networks (ICICN), 135-139. https://doi.org/10.1109/ICICN.2019.8834945

[2] Siddiqui, A., Memon, K.A., Hussain Mohammadani, K., Memon, S., Hussain M. and Abbas, M. (2021) High Order Dual Polarization Modulation Formats for Coherent Optical Systems. 2021 IEEE 11 th International Conference on Electronics Information and Emergency Communication (ICEIEC), 79-82. https://doi.org/10.1109/ICEIEC51955.2021.9463834 
[3] Schmogrow, R., et al. (2012) Error Vector Magnitude as a Performance Measure for Advanced Modulation Formats. IEEE Photon. Technol. Lett., 24, 61-63. https://doi.org/10.1109/LPT.2011.2172405

[4] Sunnerud, H., Skold, M., Westlund, M. and Andrekson, P.A. (2012) Characterization of Complex Optical Modulation Formats at $100 \mathrm{~Gb} / \mathrm{s}$ and Beyond by Coherent Optical Sampling. J. Lightwave Technol., 30, 3747-3759. https://doi.org/10.1109/JLT.2012.2204957

[5] Fatadin, I. (2016) Estimation of BER from Error Vector Magnitude for Optical Coherent Systems. Photonics, 3, 21. https://doi.org/10.3390/photonics3020021

[6] IEEE Standard for Wireless LAN Medium Access Control (MAC) and Physical Layer (PHY) Specifications: High-Speed Physical Layer in the $5 \mathrm{GHz}$ Band, IEEE Standard 802.11a-1999.

[7] Shafik, R.A., Rahman, M.S. and Islam, A.H.M.R. (2006) On the Extended Relationships among EVM, BER and SNR as Performance Metrics. Proc. 4th ICECE, 2006, 408-411. https://doi.org/10.1109/ICECE.2006.355657

[8] Pfau, T., Hoffmann, S. and Noe, R. (2009) Hardware-Efficient Coherent Digital Receiver Concept With Feedforward Carrier Recovery for $M$-QAM Constellations. J. Lightwave Technol., 27, 989-999. https://doi.org/10.1109/JLT.2008.2010511

[9] Taylor, M.G. (2009) Phase Estimation Methods for Optical Coherent Detection Using Digital Signal Processing. J. Lightwave Technol., 27, 901-914.

https://doi.org/10.1109/JLT.2008.927778

[10] Arslan, H. and Mahmoud, H.A. (2009) Error Vector Magnitude to SNR Conversion for Nondata-Aided Receivers. IEEE Trans. Wireless Commun., 8, 2694-2704. https://doi.org/10.1109/TWC.2009.080862

[11] Weber, W. (1978) Differential Encoding for Multiple Amplitude and Phase Shift Keying Systems. IEEE Transactions on Communications, 26, 385-391. https://doi.org/10.1109/TCOM.1978.1094074 